\title{
Erratum
}

\section{Erratum to: Partial Regularity of Suitable Weak Solutions to the Fractional Navier-Stokes Equations}

\author{
Lan Tang ${ }^{1}$, Yong $\mathbf{Y u}^{2}$ \\ 1 Institute of Mathematics, Academia Sinica, Taipei, Taiwan. E-mail: lantang@math.sinica.edu.tw \\ 2 Department of Mathematics, The Chinese University of Hong Kong, Shatin, Hong Kong. \\ E-mail: yongyu@math.cuhk.edu.hk
}

Received: 6 November 2014 / Accepted: 18 November 2014

Published online: 20 February 2015 - (C) Springer-Verlag Berlin Heidelberg 2015

Commun. Math. Phys. 334, 1455-1482 (2015)

We wish to correct some notations and results contained in the original article. The main results (Theorems 1.2-1.3) remain unchanged but Lemma 2.1 should be slightly corrected. ${ }^{1}$ Let us start from the corrections for some notations and typos.

(I). On Page 3 of original article, we defined the cylinders $\mathrm{Q}_{r}\left(x_{0}, t_{0}\right), \mathrm{Q}_{r}^{*}\left(x_{0}, t_{0}\right)$, $\mathrm{cQ}_{r}\left(x_{0}, t_{0}\right)$ and $\mathrm{cQ}_{r}^{*}\left(x_{0}, t_{0}\right)$. The center of these cylinders are at $\left(x_{0}, t_{0}\right)$. In fact, we need $\left(x_{0}, t_{0}\right)$ to be located on the top of these cylinders. Therefore the definitions of $\mathrm{Q}_{r}\left(x_{0}, t_{0}\right), \mathrm{Q}_{r}^{*}\left(x_{0}, t_{0}\right), \mathrm{cQ}_{r}\left(x_{0}, t_{0}\right)$ and $\mathrm{cQ}_{r}^{*}\left(x_{0}, t_{0}\right)$ on page 3 of original article should be replaced by what is shown below:

$$
\begin{aligned}
& \mathrm{Q}_{r}\left(x_{0}, t_{0}\right)=\mathrm{B}_{r}\left(x_{0}\right) \times\left(t_{0}-r^{2 s}, t_{0}\right), \quad \mathrm{Q}_{r}^{*}\left(x_{0}, t_{0}\right)=\mathrm{B}_{r}\left(x_{0}\right) \times(0, r) \times\left(t_{0}-r^{2 s}, t_{0}\right), \\
& \mathrm{cQ}_{r}\left(x_{0}, t_{0}\right)=\mathrm{cB}_{r}\left(x_{0}\right) \times\left(t_{0}-\mathrm{c}^{2 s} r^{2 s}, t_{0}\right), \quad \mathrm{cQ}_{r}^{*}\left(x_{0}, t_{0}\right)=\mathrm{cQ}_{r,+}\left(x_{0}\right) \times\left(t_{0}-\mathrm{c}^{2 s} r^{2 s}, t_{0}\right) .
\end{aligned}
$$

Moreover, the time interval $\mathrm{I}_{r}$ in Lemma 2.4 should be $\mathrm{I}_{r}=\left[-r^{2 s}, 0\right]$.

(II). In part (3) of Definition 1.1, it should be non-negative smooth function $\psi(x, t)$. There should be no variable $\mathrm{y}$ in the test function $\psi$. In (1.3), when we integrate over $\mathbb{R}_{+}^{4}, \psi$ should be understood as any function in $\mathrm{H}^{1}\left(\mathbb{R}_{+}^{4}, \mathrm{y}^{a}\right)$ which equals $\psi$ on $\mathbb{R}^{3} \times$ $\{\mathrm{y}=0\}$.

(III). In the proof of Lemma 2.6, particularly the first inequality in the proof of Lemma 2.6, the integration on the left should be over $3 / 4 \mathrm{Q}_{\rho / 3}$. The average $u_{\rho}$ in the integrand should be $u_{\rho / 3}$. Moreover, all $u_{\rho}$ in (2.18) should be $u_{\rho / 3}$. Therefore, in the estimate below (2.18), $u_{\rho}$ on the left should also be $u_{\rho / 3}$.

${ }^{1}$ We would like to express our sincere gratitude to Wei Ren at the School of Mathematics and Systems Science, BUAA, for calling our attention to a gap in the estimate of A.4 in the proof of Lemma 2.1 in original article.

The online version of the original article can be found under doi:10.1007/s00220-014-2149-z. 
(IV). In Sect. 3, centers of our cylinders should be located at possible singular points. In order to avoid ambiguity with $\mathrm{Q}_{r}(x, t)$ and $\mathrm{Q}_{r}^{*}(x, t)$ used in Sect. 2, we use the following new notations $\widetilde{\mathrm{Q}}_{r_{x, t}}^{*}(x, t)$ and $\widetilde{\mathrm{Q}}_{r_{x, t}}(x, t)$ to replace all $\mathrm{Q}_{r_{x, t}}^{*}(x, t)$ and $\mathrm{Q}_{r_{x, t}}(x, t)$ in Sect. 3, respectively. Moreover $\widetilde{\mathrm{Q}}_{r_{x_{i}, t_{i}}}$ and $\widetilde{\mathrm{Q}}_{r_{x_{i}, t_{i}}}^{*}$ should be used to replace $\mathrm{Q}_{r_{x_{i}, t_{i}}}$ and $\mathrm{Q}_{r_{x_{i}, t_{i}}^{*}}$ in Sect. 3, respectively. Therefore, in the statement of Theorem 1.2, the cylinders $\mathrm{Q}_{r}^{*}\left(x_{0}, t_{0}\right)$ should also be replaced by $\widetilde{\mathrm{Q}}_{r}^{*}\left(x_{0}, t_{0}\right)$. One should notice that if

$$
\limsup _{r \rightarrow 0^{+}} r^{4 s-5} \int_{\widetilde{\mathrm{Q}}_{r}^{*}\left(x_{0}, t_{0}\right)} \mathrm{y}^{a}\left|\bar{\nabla} u^{*}\right|^{2}
$$

is small, then obviously so is

$$
\limsup _{r \rightarrow 0^{+}} r^{4 s-5} \int_{\mathrm{Q}_{r}^{*}\left(x_{0}, t_{0}\right)} \mathrm{y}^{a}\left|\bar{\nabla} u^{*}\right|^{2} .
$$

Here $\mathrm{Q}_{r}^{*}\left(x_{0}, t_{0}\right)$ in the above is the cylinder with $\left(x_{0}, t_{0}\right)$ located on the top of it. Hence all the arguments in Sect. 2 can be applied and Proposition 2.9 holds. Using the Lebesgue differentiation theorem, we know that $\left(x_{0}, t_{0}\right)$ is a regular point.

(V). Now we begin to correct Lemma 2.1 in original article and its proof. The reason for the corrections below is due to an incorrect estimate on the term A.4 in the original proof.

Lemma 1.1 (Corrected Lemma 2.1 in original article). For any given $\left(x_{0}, t_{0}\right) \in \mathbb{R}^{3} \times \mathbb{R}_{+}$ and $k_{0} \in \mathbb{N}$, it holds

$$
\begin{aligned}
& \sup _{-r_{k}^{2 s} \leq t \leq 0} f_{\mathrm{B}^{k}\left(x_{0}\right)}|u|^{2}+r_{k}^{-3} \int_{\mathrm{Q}_{*}^{k}\left(x_{0}, t_{0}\right)} \mathrm{y}^{a}\left|\bar{\nabla} u^{*}\right|^{2} \\
& \quad \leq s r_{k_{0}}^{-5} \int_{\mathrm{Q}_{*}^{k_{0}}\left(x_{0}, t_{0}\right)} \mathrm{y}^{a}\left|u^{*}\right|^{2}+f_{\mathrm{Q}^{k_{0}}\left(x_{0}, t_{0}\right)}|u|^{2}+r_{k_{0}}^{-3} \int_{\mathrm{Q}_{*}^{k_{0}}} \mathrm{y}^{a}\left|\bar{\nabla} u^{*}\right|^{2} \\
& \quad+\sum_{l=k_{0}}^{k} r_{l}^{2 s} f_{\mathrm{Q}^{l}\left(x_{0}, t_{0}\right)}|f u|+\sum_{l=k_{0}}^{k} r_{l}^{-a} f_{\mathrm{Q}^{l}\left(x_{0}, t_{0}\right)}|u|^{3}+|u| \cdot\left|p-\bar{p}_{l}\right|, \quad \forall k>k_{0},
\end{aligned}
$$

where $\bar{p}_{l}$ is the average of $p$ over the ball $\mathrm{B}^{l}\left(x_{0}\right)$ in $\mathbb{R}^{3}$.

Proof. The test functions $\chi_{1}, \chi_{2}$ and $\chi$ are the same as in the proof of Lemma 2.1 in original article. $\mathrm{Q}^{k}\left(x_{0}, t_{0}\right)$ are cylinders with $\left(x_{0}, t_{0}\right)$ located on the top of them. Without loss of generality, we assume $\left(x_{0}, t_{0}\right)=(0,0)$ in the following arguments. Here $\phi_{k}$ is different from what is used in original article and we choose $\phi_{k}$ to be the backward heat kernel of $(-\Delta)^{s}$ on $\mathbb{R}^{3}$. Using the same notation as in [1], $\phi_{k}$ can be written as:

$$
\phi_{k}(t, x)=p_{2 s}\left(r_{k}^{2 s}-t, x\right) \text {. }
$$

Then we insert $\psi=\chi_{1} \phi_{k}$ into (1.3) of original article. It should be mentioned that when we take the integral over $\mathbb{R}_{+}^{4}, \psi$ should be understood as $\chi \phi_{k}^{*}$, where $\phi_{k}^{*}$ is the Caffarelli-Silvestre extension of $\phi_{k}$. Then we have 


$$
\begin{aligned}
& \int_{\mathbb{R}^{3} \times\{t\}}|u|^{2} \psi+2 \mathrm{C}_{s} \int_{-r_{k_{0}}^{2 s}}^{t} \int_{\mathbb{R}_{+}^{4}} \psi \mathrm{y}^{a}\left|\bar{\nabla} u^{*}\right|^{2} \\
& \leq \mathrm{C}_{s} \int_{-r_{k_{0}}^{2 s}}^{t} \int_{\mathbb{R}_{+}^{4}}\left|u^{*}\right|^{2} \operatorname{Div}\left(\mathrm{y}^{a} \bar{\nabla} \psi\right)+\int_{-r_{k_{0}}^{2 s}}^{t} \int_{\mathbb{R}^{3}}(u \cdot \nabla \psi)\left(2 p+|u|^{2}\right) \\
& \quad+\int_{-r_{k_{0}}^{2 s}}^{t} \int_{\mathbb{R}^{3}}|u|^{2}\left[\psi_{t}+\mathrm{C}_{s} \lim _{\mathrm{y} \rightarrow 0^{+}}\left(\mathrm{y}^{a} \partial_{\mathrm{y}} \psi\right)\right]+2 \int_{-r_{k_{0}}^{2 s}}^{t} \int_{\mathbb{R}^{3}} \psi f \cdot u \quad \forall t \in\left[-r_{k_{0}}^{2 s}, 0\right] .
\end{aligned}
$$

For convenience we denote the four terms on the right-hand side of (1.1) by A, B, C and $\mathrm{D}$, respectively.

(1) The term A can be split into three parts: $\mathrm{A}=\mathrm{A} .1+\mathrm{A} .2+\mathrm{A} .3$, where

$$
\begin{aligned}
& \mathrm{A} .1=a \mathrm{C}_{s} \int_{-r_{k_{0}}^{2 s}}^{t} \int_{\mathbb{R}_{+}^{4}} \mathrm{y}^{a}\left|u^{*}\right|^{2} \mathrm{y}^{-1} \chi_{1} \chi_{2}^{\prime} \phi_{k}^{*} ; \\
& \mathrm{A} .2=\mathrm{C}_{s} \int_{-r_{k_{0}}^{2 s}}^{t} \int_{\mathbb{R}_{+}^{4}} \mathrm{y}^{a}\left|u^{*}\right|^{2} \phi_{k}^{*} \operatorname{Div}(\bar{\nabla} \chi) ; \quad \mathrm{A} .3=2 \mathrm{C}_{s} \int_{-r_{k_{0}}^{2 s}}^{t} \int_{\mathbb{R}_{+}^{4}} \mathrm{y}^{a}\left|u^{*}\right|^{2} \bar{\nabla} \chi \cdot \bar{\nabla} \phi_{k}^{*} .
\end{aligned}
$$

Here we have used Div $\left(\mathrm{y}^{a} \bar{\nabla} \phi_{k}^{*}\right)=0$.

Estimate for A.1. By Poisson kernel, $\phi_{k}^{*}$ can be represented by

$$
\phi_{k}^{*}(x, \mathrm{y}, t)=\int_{\mathbb{R}^{3}} \frac{\mathrm{y}^{2 s}}{\left(|x-\xi|^{2}+\mathrm{y}^{2}\right)^{\frac{3+2 s}{2}}} \phi_{k}(\xi, t) \mathrm{d} \xi,
$$

which, together with (1.12) in [1], shows that

$$
\phi_{k}^{*}(x, \mathrm{y}, t) \lesssim s r_{k_{0}}^{-3} \int_{\mathbb{R}^{3}} \phi_{k}(\xi, t)=r_{k_{0}}^{-3}, \quad \forall \mathrm{y} \in\left[r_{k_{0}+1}, r_{k_{0}}\right] .
$$

Since $\operatorname{supp}\left(\chi_{2}^{\prime}\right) \subset\left[r_{k_{0}+1}, r_{k_{0}}\right]$ and $\left\|\chi_{2}^{\prime}\right\|_{\mathrm{L}^{\infty}(\mathbb{R})} \lesssim r_{k_{0}}^{-1}$, then by (1.3), A.1 can be estimated by

$$
|\mathrm{A} .1| \lesssim s \int_{\mathrm{Q}_{*}^{k_{0}}} \mathrm{y}^{a}\left|u^{*}\right|^{2} \mathrm{y}^{-1} \chi_{1}\left|\chi_{2}^{\prime}\right| \phi_{k}^{*} \lesssim s r_{k_{0}}^{-5} \int_{\mathrm{Q}_{*}^{k_{0}}} \mathrm{y}^{a}\left|u^{*}\right|^{2} .
$$

Estimate for A.2. By direct computation, we know that $\operatorname{Div}(\bar{\nabla} \chi)=\chi_{2} \Delta \chi_{1}+\chi_{1} \chi_{2}^{\prime \prime}$. In light of the facts that $\operatorname{supp}\left(\chi_{2}^{\prime \prime}\right) \subset\left[r_{k_{0}+1}, r_{k_{0}}\right]$ and $\left\|\chi_{2}{ }^{\prime \prime}\right\|_{L^{\infty}(\mathbb{R})} \leq r_{k_{0}}^{-2}$, we have, by (1.3), that

$$
\left.\left.\left|\int_{\mathrm{Q}_{*}^{k_{0}}} \mathrm{y}^{a}\right| u^{*}\right|^{2} \phi_{k}^{*} \chi_{1} \chi_{2}^{\prime \prime}\left|\lesssim s r_{k_{0}}^{-5} \int_{\mathrm{Q}_{*}^{k_{0}}} \mathrm{y}^{a}\right| u^{*}\right|^{2}
$$

By (1.2), we know that

$$
\begin{aligned}
\phi_{k}^{*}(x, \mathrm{y}, t) & =\int_{\mathrm{B}^{k_{0}+2}} \frac{\mathrm{y}^{2 s}}{\left(|x-\xi|^{2}+\mathrm{y}^{2}\right)^{\frac{3+2 s}{2}}} \phi_{k}(\xi, t)+\int_{\mathbb{R}^{3} \backslash \mathrm{B}^{k_{0}+2}} \frac{\mathrm{y}^{2 s}}{\left(|x-\xi|^{2}+\mathrm{y}^{2}\right)^{\frac{3+2 s}{2}}} \phi_{k}(\xi, t) \\
& \lesssim s r_{k_{0}}^{-3} \int_{\mathrm{B}^{k_{0}+2}} \phi_{k}(\xi, t)+\int_{\mathbb{R}^{3} \backslash \mathrm{B}^{k_{0}+2}} \frac{\mathrm{y}^{2 s}}{\left(|x-\xi|^{2}+\mathrm{y}^{2}\right)^{\frac{3+2 s}{2}}} \frac{r_{k}^{2 s}-t}{\left[\left(r_{k}^{2 s}-t\right)^{1 /(2 s)}+|\xi|\right]^{3+2 s}} \\
& \lesssim s r_{k_{0}}^{-3}, \quad \text { provided that }|x|>r_{k_{0}+1}, \mathrm{y} \in\left(0, r_{k_{0}}\right) \text { and }|t| \leq r_{k_{0}}^{2 s} .
\end{aligned}
$$


Here we have used the following facts: (i) $|x-\xi|>r_{k_{0}+2}$ with $|x|>r_{k_{0}+1}$ and $|\xi|<$ $r_{k_{0}+2}$; (ii) the estimates (1.3) and (1.12) in [1]. Since $\operatorname{supp}\left(\Delta \chi_{1}\right) \subset \mathrm{B}^{k_{0}} \backslash \mathrm{B}^{k_{0}+1}$ and $\left\|\Delta \chi_{1}\right\|_{L^{\infty}\left(\mathbb{R}^{3}\right)} \lesssim r_{k_{0}}^{-2}$, we have, by (1.6), that

$$
\left.\left.\left|\int_{\mathrm{Q}_{*}^{k_{0}}} \mathrm{y}^{a}\right| u^{*}\right|^{2} \phi_{k}^{*} \chi_{2} \Delta \chi_{1}\left|\lesssim s r_{k_{0}}^{-5} \int_{\mathrm{Q}_{*}^{k_{0}}} \mathrm{y}^{a}\right| u^{*}\right|^{2},
$$

which, together with (1.5), shows that

$$
|\mathrm{A} .2| \lesssim s r_{k_{0}}^{-5} \int_{\mathrm{Q}_{*}^{k_{0}}} \mathrm{y}^{a}\left|u^{*}\right|^{2} .
$$

Estimate for A.3. By the formula (1.2), we know that

$$
\nabla \phi_{k}^{*}(x, \mathrm{y}, t)=\int_{\mathbb{R}^{3}} \frac{\mathrm{y}^{2 s}(x-\xi)}{\left(|x-\xi|^{2}+\mathrm{y}^{2}\right)^{\frac{3+2 s}{2}+1}}\left(\phi_{k}(\xi, t)-\phi_{k}(x, t)\right) \mathrm{d} \xi .
$$

Since $u^{*}(x, \mathrm{y})=u(x)+\int_{0}^{\mathrm{y}} \partial_{z} u^{*} \mathrm{~d} z$, therefore it holds by (1.8) and Hölder's inequality that

$$
\begin{aligned}
\left.\left|\int_{\mathrm{Q}_{*}^{k_{0}}}\right| u^{*}\right|^{2} \mathrm{y}^{a} \nabla \chi \cdot \nabla \phi_{k}^{*} \mid \lesssim & \int_{\mathrm{Q}_{*}^{k_{0}}}\left|\int_{0}^{\mathrm{y}} \partial_{z} u^{*}\right|^{2} \mathrm{y}^{a}|\nabla \chi| \int_{\mathbb{R}^{3}} \frac{\mathrm{y}^{2 s}|x-\xi|\left|\phi_{k}(\xi, t)-\phi_{k}(x, t)\right|}{\left(|x-\xi|^{2}+\mathrm{y}^{2}\right)^{\frac{3+2 s}{2}+1} \mathrm{~d} \xi} \\
& +\int_{\mathrm{Q}_{*}^{k_{0}}}|u|^{2} \mathrm{y}^{a}|\nabla \chi| \int_{\mathbb{R}^{3}} \frac{\mathrm{y}^{2 s}|x-\xi|\left|\phi_{k}(\xi, t)-\phi_{k}(x, t)\right|}{\left(|x-\xi|^{2}+\mathrm{y}^{2}\right)^{\frac{3+2 s}{2}+1} \mathrm{~d} \xi} \\
\lesssim & r_{k_{0}}^{2 s} \int_{\mathrm{Q}_{*}^{k_{0}}} \int_{0}^{r_{k_{0}}} z^{a}\left|\bar{\nabla} u^{*}\right|^{2}|\nabla \chi| \int_{\mathbb{R}^{3}} \frac{\mathrm{y}|x-\xi|\left|\phi_{k}(\xi, t)-\phi_{k}(x, t)\right|}{\left(|x-\xi|^{2}+\mathrm{y}^{2}\right)^{\frac{3+2 s}{2}+1}} \mathrm{~d} \xi \\
& +\int_{\mathrm{Q}_{*}^{k_{0}}}|u|^{2}|\nabla \chi| \int_{\mathbb{R}^{3}} \frac{\mathrm{y}|x-\xi|\left|\phi_{k}(\xi, t)-\phi_{k}(x, t)\right|}{\left(|x-\xi|^{2}+\mathrm{y}^{2}\right)^{\frac{3+2 s}{2}+1} \mathrm{~d} \xi}
\end{aligned}
$$

Integrating the variable y from 0 to $r_{k_{0}}$ in the last two integrals above, we can get

$$
\begin{aligned}
\left.\left|\int_{\mathrm{Q}_{*}^{k_{0}}}\right| u^{*}\right|^{2} \mathrm{y}^{a} \nabla \chi \cdot \nabla \phi_{k}^{*} \mid \lesssim s & r_{k_{0}}^{2 s} \int_{\mathrm{Q}^{k_{0}}} \int_{0}^{r_{k_{0}}} z^{a}\left|\bar{\nabla} u^{*}\right|^{2}\left|\nabla \chi_{1}\right| \int_{\mathbb{R}^{3}} \frac{\left|\phi_{k}(\xi, t)-\phi_{k}(x, t)\right|}{|x-\xi|^{2+2 s}} \\
& +\int_{\mathrm{Q}^{k_{0}}}|u|^{2}\left|\nabla \chi_{1}\right| \int_{\mathbb{R}^{3}} \frac{\left|\phi_{k}(\xi, t)-\phi_{k}(x, t)\right|}{|x-\xi|^{2+2 s}}
\end{aligned}
$$

By the definition of $\chi_{1}$, we know that $\operatorname{supp}\left(\nabla \chi_{1}\right) \subset \mathrm{B}^{k_{0}} \backslash \mathrm{B}^{k_{0}+1}$ and $\left\|\nabla \chi_{1}\right\|_{\mathrm{L}^{\infty}\left(\mathbb{R}^{3}\right)} \leq r_{k_{0}}^{-1}$. Hence the integration on the right-hand side of (1.9) is over the set where $|x|$ ranges from $r_{k_{0}+1}$ to $r_{k_{0}}$.

Case 1. If $|\xi| \leq r_{k_{0}+2}$ or $|\xi| \geq r_{k_{0}-1}$, then $|x-\xi| \geq r_{k_{0}+2}$. Meanwhile, by (1.3) in [1], we have that $\phi_{k}(x, t) \lesssim s r_{k_{0}}^{-3}$ if $|x| \geq r_{k_{0}+1}$ and $|t| \leq r_{k_{0}}^{2 s}$. Therefore, it holds

$$
\begin{aligned}
& \int_{|\xi| \leq r_{k_{0}+2}} \frac{\left|\phi_{k}(\xi, t)-\phi_{k}(x, t)\right|}{|x-\xi|^{2+2 s}}+\int_{|\xi| \geq r_{k_{0}-1}} \frac{\left|\phi_{k}(\xi, t)-\phi_{k}(x, t)\right|}{|x-\xi|^{2+2 s}} \\
& \lesssim s r_{k_{0}}^{-2-2 s} \int_{\mathbb{R}^{3}} \phi_{k}(\xi, t)+\phi_{k}(x, t) \int_{|x-\xi| \geq r_{k_{0}+2}} \frac{1}{|x-\xi|^{2+2 s}} \lesssim s r_{k_{0}}^{-2-2 s} .
\end{aligned}
$$


Case 2. If $r_{k_{0}+2} \leq|\xi| \leq r_{k_{0}-1}$, then $|x-\xi|<r_{k_{0}-2}$. By (1.9) in [1], we know that

$$
\begin{aligned}
\int_{r_{k_{0}+2}|\leq| \xi \mid \leq r_{k_{0}+1}} \frac{\left|\phi_{k}(\xi, t)-\phi_{k}(x, t)\right|}{|x-\xi|^{2+2 s}} & \lesssim s \int_{r_{k_{0}+2}|\leq| \xi \mid \leq r_{k_{0}+1}} \frac{\left(r_{k}^{2 s}-t\right)^{1-\frac{\gamma}{2 s}}(|\xi| \wedge|x|)^{-3-2 s}}{|x-\xi|^{2+2 s-\gamma}} \\
& \lesssim s r_{k_{0}}^{-3-\gamma} \int_{|x-\xi|<r_{k_{0}-2}} \frac{1}{|x-\xi|^{2+2 s-\gamma}} \lesssim s r_{k_{0}}^{-2-2 s},
\end{aligned}
$$

where $\gamma \in(2 s-1,1)$ is a constant. Applying (1.10)-(1.11) to (1.9), we have

$$
\left.\left.\left|\int_{\mathrm{Q}_{*}^{k_{0}}}\right| u^{*}\right|^{2} \mathrm{y}^{a} \nabla \chi \cdot \nabla \phi_{k}^{*}\left|\lesssim s r_{k_{0}}^{-3} \int_{\mathrm{Q}_{*}^{k_{0}}} \mathrm{y}^{a}\right| \bar{\nabla} u^{*}\right|^{2}+r_{k_{0}}^{-3-2 s} \int_{\mathrm{Q}^{k_{0}}}|u|^{2} .
$$

In the following we complete the estimate for A. 3 by studying

$$
\int_{\mathrm{Q}_{*}^{k_{0}}}\left|u^{*}\right|^{2} \mathrm{y}^{a} \chi_{1} \chi_{2}^{\prime} \partial_{\mathrm{y}} \phi_{k}^{*}
$$

By the formula (1.2), we have

$$
\partial_{\mathrm{y}} \phi_{k}^{*}=\int_{\mathbb{R}^{3}} \frac{2 s|x-\xi|^{2} \mathrm{y}^{2 s-1}-3 \mathrm{y}^{2 s+1}}{\left(|x-\xi|^{2}+\mathrm{y}^{2}\right)^{\frac{3+2 s}{2}+1}} \phi_{k}(\xi, t) .
$$

Since $y \in\left[r_{k_{0}+1}, r_{k_{0}}\right]$, we can easily show that

$$
\begin{aligned}
\left.\left|\int_{\mathrm{Q}_{*}^{k_{0}}}\right| u^{*}\right|^{2} \mathrm{y}^{a} \chi_{1} \chi_{2}^{\prime} \partial_{\mathrm{y}} \phi_{k}^{*} \mid \lesssim s & r_{k_{0}}^{-5} \int_{\mathrm{Q}_{*}^{k_{0}}} \mathrm{y}^{a}\left|u^{*}\right|^{2} \\
& +\int_{\mathrm{Q}_{*}^{k_{0}}}\left|u^{*}\right|^{2} \mathrm{y}^{a}\left|\chi_{2}^{\prime}\right| \int_{\mathbb{R}^{3}} \frac{|x-\xi|^{2} \mathrm{y}^{2 s-1} \phi_{k}(\xi, t)}{\left(|x-\xi|^{2}+\mathrm{y}^{2}\right)^{\frac{3+2 s}{2}+1}} .
\end{aligned}
$$

If $|\xi|>r_{k_{0}-1}$, then $|x-\xi| \geq r_{k_{0}}$. Therefore, using (1.3) in [1], we have

$$
\int_{|\xi|>r_{k_{0}-1}} \frac{|x-\xi|^{2} \mathrm{y}^{2 s-1} \phi_{k}(\xi, t)}{\left(|x-\xi|^{2}+\mathrm{y}^{2}\right)^{\frac{3+2 s}{2}+1}} \lesssim s r_{k_{0}}^{2 s-4} \int_{|x-\xi| \geq r_{k_{0}}} \frac{1}{|x-\xi|^{3+2 s}} \lesssim s r_{k_{0}}^{-4} .
$$

If $|\xi| \leq r_{k_{0}-1}$, then $|x-\xi| \leq r_{k_{0}-2}$. Since $\mathrm{y} \in\left[r_{k_{0}+1}, r_{k_{0}}\right]$, therefore it holds

$$
\int_{|\xi| \leq r_{k_{0}-1}} \frac{|x-\xi|^{2} \mathrm{y}^{2 s-1} \phi_{k}(\xi, t)}{\left(|x-\xi|^{2}+\mathrm{y}^{2}\right)^{\frac{3+2 s}{2}+1}} \lesssim s r_{k_{0}}^{-4} \int_{\mathbb{R}^{3}} \phi_{k}(\xi, t)=r_{k_{0}}^{-4} .
$$

Applying the last two estimates above to (1.13), we get

$$
\left.\left.\left|\int_{\mathrm{Q}_{*}^{k_{0}}}\right| u^{*}\right|^{2} \mathrm{y}^{a} \chi_{1} \chi_{2}^{\prime} \partial_{\mathrm{y}} \phi_{k}^{*}\left|\lesssim_{s} r_{k_{0}}^{-5} \int_{\mathrm{Q}_{*}^{k_{0}}} \mathrm{y}^{a}\right| u^{*}\right|^{2},
$$

which, together with (1.12), completes the estimate for A.3.

(2) The estimates for terms B and D are the same as those in the proof of Lemma 2.1 of our original article. However, one should notice that B.1 defined in the proof of Lemma 2.1 in original article is incorrect. It should be 


$$
\mathrm{B} .1=\int_{\mathrm{Q}^{k_{0}}}|u|^{2} u \cdot \nabla \psi_{k} .
$$

Moreover, when there is a need to estimate $\phi_{k}$ and $\nabla \phi_{k}$, we refer readers to (1.3) and (1.15) in [1].

(3) The term $\mathrm{C}$ is given by

$$
\mathrm{C}=\int_{\mathrm{Q}^{k_{0}}}|u|^{2}\left\{\partial_{t} \psi+\mathrm{C}_{s} \lim _{\mathrm{y} \rightarrow 0^{+}}\left(\mathrm{y}^{a} \partial_{\mathrm{y}} \psi\right)\right\} .
$$

Since $\phi_{k}$ is the backward heat kernel of $(-\Delta)^{s}$, therefore it holds

$$
\partial_{t} \psi+\mathrm{C}_{s} \lim _{\mathrm{y} \rightarrow 0^{+}} \mathrm{y}^{a} \partial_{\mathrm{y}} \psi=\phi_{k} \partial_{t} \chi_{1}+\chi_{1} \partial_{t} \phi_{k}+\chi_{1} \mathrm{C}_{s} \lim _{\mathrm{y} \rightarrow 0^{+}} \mathrm{y}^{a} \partial_{\mathrm{y}} \phi_{k}^{*}=\phi_{k} \partial_{t} \chi_{1},
$$

which shows that

$$
\mathrm{C}=\int_{\mathrm{Q}^{k_{0}}}|u|^{2} \partial_{t} \chi_{1} \phi_{k}
$$

One can easily have (2.7) in original article by (1.3) in [1].

(4) Now we consider the lower bound for the left-hand side of (1.1). Fixing a $t \in$ $\left[-r_{k}^{2 s}, 0\right]$, we have

$$
\int_{\mathbb{R}^{3} \times\{t\}}|u|^{2} \psi \geq \int_{\mathrm{B}^{k} \times\{t\}}|u|^{2} \phi_{k}, \quad \forall k>k_{0} .
$$

Using (1.3) in [1], we can easily show that

$\int_{\mathrm{B}^{k} \times\{t\}}|u|^{2} \phi_{k} \gtrsim s \int_{\mathrm{B}^{k} \times\{t\}}|u|^{2} \frac{r_{k}^{2 s}-t}{\left(\left(r_{k}^{2 s}-t\right)^{1 /(2 s)}+|x|\right)^{3+2 s}} \gtrsim s r_{k}^{-3} \int_{\mathrm{B}^{k} \times\{t\}}|u|^{2}, \quad \forall k>k_{0}$.

Here we have used the facts that $|x| \leq r_{k}$ and $-r_{k}^{2 s} \leq t \leq 0$. Therefore, the estimate in Lemma 1.1 on the average of $|u|^{2}$ over $\mathrm{B}^{k}$ is obtained since the last estimate holds for all $t \in\left[-r_{k}^{2 s}, 0\right]$.

In the end, we estimate the lower bound of the integral

$$
\int_{\mathrm{Q}_{*}^{k_{0}}} \psi \mathrm{y}^{a}\left|\bar{\nabla} u^{*}\right|^{2}
$$

For all $k>k_{0}$, we have $\mathrm{Q}_{*}^{k} \subset \mathrm{Q}_{*}^{k_{0}}$, which shows

$$
\int_{\mathrm{Q}_{*}^{k_{0}}} \psi \mathrm{y}^{a}\left|\bar{\nabla} u^{*}\right|^{2} \geq \int_{\mathrm{Q}_{*}^{k}} \phi_{k}^{*} \mathrm{y}^{a}\left|\bar{\nabla} u^{*}\right|^{2} .
$$

From (1.2), we know that

$$
\phi_{k}^{*}(x, \mathrm{y}, t) \geq \int_{\{\xi:|x-\xi|<\mathrm{y}\}} \frac{\mathrm{y}^{2 s} \phi_{k}(\xi, t)}{\left(|x-\xi|^{2}+\mathrm{y}^{2}\right)^{\frac{3+2 s}{2}}} .
$$

If $|x-\xi|<\mathrm{y}$ with $\mathrm{y} \in\left[0, r_{k}\right]$ and $x \in \mathrm{B}^{k}$, then $|\xi| \leq r_{k-1}$. By (1.3) in [1], it holds

$$
\begin{aligned}
\phi_{k}(\xi, t) & \gtrsim s \quad\left(r_{k}^{2 s}-t\right)\left(\left(r_{k}^{2 s}-t\right)^{1 /(2 s)}+|\xi|\right)^{-3-2 s} \\
& \gtrsim s \quad r_{k}^{-3}, \quad \text { provided that }|\xi| \leq r_{k-1} \text { and } t \in\left[-r_{k}^{2 s}, 0\right] .
\end{aligned}
$$


Therefore, the last two estimates above indicate that

$$
\begin{aligned}
\phi_{k}^{*}(x, \mathrm{y}, t) & \gtrsim s r_{k}^{-3} \int_{\{\xi:|x-\xi|<\mathrm{y}\}} \frac{\mathrm{y}^{2 s}}{\left(|x-\xi|^{2}+\mathrm{y}^{2}\right)^{\frac{3+2 s}{2}}} \\
& \gtrsim s r_{k}^{-3} \mathrm{y}^{-3}\left|\mathrm{~B}_{\mathrm{y}}(x)\right|=\frac{4 \pi}{3} r_{k}^{-3}, \quad \forall(x, \mathrm{y}, t) \in \mathrm{Q}_{*}^{k} .
\end{aligned}
$$

Applying (1.15) to (1.14), we finally have

$$
\int_{\mathrm{Q}_{*}^{k_{0}}} \psi \mathrm{y}^{a}\left|\bar{\nabla} u^{*}\right|^{2} \gtrsim s r_{k}^{-3} \int_{\mathrm{Q}_{*}^{k}} \mathrm{y}^{a}\left|\bar{\nabla} u^{*}\right|^{2} .
$$

The proof is then finished.

\section{Reference}

1. Chen, Z.Q., Zhang, X.: Heat kernels and analyticity of non-symmetric jump diffusion semigroups. arXiv: $1306.5015 \mathrm{v} 2$

Communicated by L. Caffarelli 